\title{
Epoxy-Acrylic Core-Shell Particles by Seeded Emulsion Polymerization
}

Liang Chen, ${ }^{+*}$ Liang Hong, ${ }^{\dagger}$ Jui-Ching Lin, ${ }^{\#}$ Greg Meyers, ${ }^{\#}$ Joseph Harris, ${ }^{\#}$ Michael Radler ${ }^{ \pm}$

$\dagger$ Formulation Science, The Dow Chemical Company, Midland, MI 48674

\# Analytical Sciences, The Dow Chemical Company, Midland, MI 48674

\pm Dow Construction Chemicals, The Dow Chemical Company, Midland, MI 48674

*Author to whom correspondence should be addressed. E-mail: LiangChen@Dow.com 


\section{Keywords:}

Mechanical dispersion, Seeded emulsion polymerization, Epoxy-acrylic core-shell particles, Scanning transmission X-ray microscopy, Peak force tapping atomic force microscopy 


\section{Introduction}

Hybrid polymer latexes with complex morphologies have been widely used in coating, construction, and adhesive applications [1,2]. Synergy between two distinct chemistries can be achieved to offer tremendous advantages in performance over an individual polymer latex or a blend of two different latexes. Hybrid polymer latexes are commonly synthesized by either a mini-emulsion polymerization [3] or seeded emulsion polymerization process [4]. There have been tremendous research efforts on the preparation of hybrid polymer latexes, such as siliconeacrylate [5], acrylate-urethane [6], styrene-acrylate [7], and PVDF-acrylic [8]. Among these examples, various particle morphologies, including the core-shell morphology, were often specifically designed [9].

We are interested in epoxy-acrylic hybrid latexes where the unique performance attributes of both chemistries can be combined. Acrylic latexes have been used in various applications owing to their tunable mechanical, aesthetic, and chemical properties, while waterborne epoxy dispersions have been applied in protective and maintenance coatings due to their benefits such as hardness and chemical resistance [10]. The mini-emulsion approach has been widely taken to synthesize the epoxy-acrylic hybrid latexes. The epoxy prepolymers are first dissolved into the acrylic monomers. The mixture is emulsified in water to form well-defined submicron droplets, and finally, the monomers are polymerized by free radical polymerization [11-13]. By this approach, the epoxy groups from the original epoxy prepolymers remain intact throughout the polymerization process. Liu and coworkers demonstrated the epoxy-acrylic hybrid latexes by a two-stage emulsion polymerization process [14], but the morphology of the resulting latexes was still ill-defined. In addition, a new method was recently developed to synthesize epoxy-acrylic hybrid latexes [15], where a liquid epoxy prepolymer was post-mixed with the acrylic latexes 
and absorbed into the acrylic particles. In all of the previous examples, only liquid epoxy prepolymers were successfully used in the synthesis of epoxy-acrylic hybrid latexes, and the epoxy prepolymers were typically limited to less than $50 \mathrm{wt} \%$ in the hybrid particles. For some applications where performance attributes from epoxy resins are strongly desired, new methods are required to broadly tune the composition of the epoxy-acrylic hybrid latexes.

In this paper, we developed a novel method for synthesizing epoxy-acrylic hybrid latexes as illustrated in Figure 1. We first prepared an aqueous dispersion of high molecular weight solid epoxy prepolymers by a continuous solvent-free mechanical dispersion process. This process was developed to create stable aqueous dispersions of polymers that cannot be produced by conventional emulsification techniques. By this process, high internal phase ratio emulsions [16] were first formed and then diluted to polymer dispersions at the desired solid content [17]. For example, high viscosity silicone polymers were processed to form very small droplet sizes with narrow particle size distributions [18]. Herein, we have successfully prepared solid epoxy particles of less than $400 \mathrm{~nm}$ with a narrow particle size distribution by using polyvinyl alcohol as a dispersant. Next, we used the epoxy dispersion as a seed in the emulsion polymerization of acrylic monomers comprising methyl methacrylate (MMA) and methacrylic acid (MAA). We characterized the morphology of the epoxy-acrylic hybrid latex particles by using both scanning transmission X-ray microscopy (STXM) and peak force tapping atomic force microscopy (PFTAFM) techniques and proved a well-defined core-shell morphology. This new approach allows us to tune the composition of the hybrid polymer latexes in two independent steps, and it can produce new epoxy-acrylic hybrid latexes potentially useful for various applications.

\section{Materials and methods}




\subsection{Materials.}

Bisphenol-A epoxy resin prepolymers [19] (D.E.R. ${ }^{\text {TM }}$ 331, D.E.R. ${ }^{\text {TM }}$ 669E) were obtained from The Dow Chemical Company. Polyvinyl alcohol (Mowiol ${ }^{\mathrm{TM}} 4-88$ ) was purchased from Kuraray. Sodium formaldehyde sulfoxylate (SFS), tert-butyl peroxide (70\% solution in water) (t-BuOOH), sodium dodecylbenzene sulfonate (SDBS), methylmethacrylate ( $>99 \%$ purity), and methacrylic acid ( $>99 \%$ purity) were obtained from Aldrich and were used without further purification.

\subsection{Synthesis of epoxy-acrylic hybrid latex}

2.2.1. Batch Dispersion Process. The experimental protocol was described in the literature [20]. A blend of D.E.R. ${ }^{\text {TM }} 331$ and D.E.R. ${ }^{\text {TM }} 669 \mathrm{E}$ in a weight ratio of 45:55 (50.0 g) and a polyvinyl alcohol (PVOH) solution (28.0 wt\% solid) $(18.0 \mathrm{~g})$ were first charged into a $300-\mathrm{mL}$ stainless steel Parr reactor equipped with a Cowles blade. The reactor was pre-heated to $100{ }^{\circ} \mathrm{C}$, and the mixture was stirred for 10 min to allow sufficient mixing of the epoxy phase and the PVOH solution at a speed of $1800 \mathrm{rpm}$. To this mixture, deionized (DI) water was added using an HPLC pump at the rate of $1.0 \mathrm{~mL} / \mathrm{min}$ over $20 \mathrm{~min}$. After that, the addition rate of DI water was increased to $10 \mathrm{~mL} / \mathrm{min}$ for an additional $4 \mathrm{~min}$. The reactor was then cooled down to $50{ }^{\circ} \mathrm{C}$ in a water bath at a mixing speed of approximately $500 \mathrm{rpm}$. The resultant dispersion was collected by filtration through a 190 micron filter.

2.2.2. Seeded Emulsion Polymerization. The experimental procedure was modified based on the protocol described in the literature [21]. A diluted epoxy dispersion (94.1 g, $40 \mathrm{wt} \%$ solids) was charged into a $250 \mathrm{~mL}$ three-neck flask fitted with a condenser and a mechanical stirrer. The flask was soaked in a water bath set at $65^{\circ} \mathrm{C}$. The stir rod was inserted through the Teflon adaptor and glass sleeve and connected to the center of the flask. The stirrer rate was set at 200 rpm. Nitrogen was slowly purged through the reactor, and cooling water was turned on to flow 
through the condenser. MMA (8.21 g) and MAA (2.05 g) were mixed with DI water (2.56 g) and SDBS $(0.020 \mathrm{~g})$ in a glass jar to form an emulsion, and the mixture was fed into the flask by a syringe pump at a steady rate over $40 \mathrm{~min}$. SFS (0.062 grams) and t-BuOOH (70\% active) $(0.088$ grams) were dissolved in $5 \mathrm{~g}$ of DI water, respectively, and then fed by two separate syringe pumps into the reactor at the same rate over $60 \mathrm{~min}$. After feeding the initiators, the reaction was held at $65{ }^{\circ} \mathrm{C}$ for one hour. Finally, the epoxy-acrylic hybrid latex was collected by filtration through a 190 micron filter. Some of the dispersion sample was dried at ambient temperature to form a powder for further analyses.

\subsection{Transmission electron microscopy (TEM)}

The polymer latex was diluted in DI water, and then, a $\sim 0.5-\mu \mathrm{L}$ drop of solution was placed onto a carbon-coated TEM grid. The dry polymer powder was embedded in BEEM capsules using D.E.R. ${ }^{\text {TM }} 330$ epoxy resin with D.E.H. ${ }^{\text {TM }} 24$ epoxy curing agent. A Leica UC6 microtome was used to collect 90-nm-thick sections on 400 mesh copper TEM grids. Sections were vapor stained over $0.5 \%$ ruthenium tetroxide for $3 \mathrm{~min}$. Samples were examined with a JEOL JEM1230 TEM operating at $120 \mathrm{kV}$. Digital images were collected with a Gatan MultiScan CCD camera.

\subsection{Scanning Transmission X-ray Microscopy (STXM)}

Scanning transmission X-ray microscopy was performed using the X-ray microscope at beamline 5.3.2.2 at the Advanced Light Source at Lawrence Berkeley National Laboratory. The instrument setup has been described elsewhere [22]. The samples were scanned in the $\mathrm{x}$ and $\mathrm{y}$ directions using piezo-ceramic positioning to build up an image on a pixel-by-pixel basis. The spatial resolution of the measurement was approximately $30 \mathrm{~nm}$. Hyperspectral images were collected from the sample by acquiring images of the same area at multiple energies providing one 

optical density image stack using Axis 2000 program [23]. The ratio of the epoxy to acrylic polymers was determined by performing a non-negative least square fitting (NNLS) on the NEXAFS spectrum pixel by pixel using a Matlab program developed in-house, assuming the spectrum at each pixel is a linear combination of the pure component spectra collected from the pure epoxy and acrylic polymers used in this study (Figure 2) with a non-negative concentration. The sample was prepared by drying a drop of diluted polymer latex on a thin silicon nitride window. The polymer latex used for the STXM characterization had a slightly different epoxy composition (DER669E:DER331=1:1 by weight) than the sample shown in the rest of the paper.

\subsection{Atomic force microscopy (AFM)}

The dried powder was embedded into BEEM capsules using D.E.R. ${ }^{\text {TM }} 330$ epoxy resin with D.E.H. ${ }^{\text {TM }} 24$ epoxy curing agent. A Leica UC6 microtome was used to collect $150 \mathrm{~nm}$ thin sections on cleaved mica. AFM images were collected using two methods: conventional tapping mode (TMAFM) and peak force tapping (PFT). Tapping mode AFM is a resonant cantilever imaging technique that provides both topographic and phase lag imaging, the latter of which is useful for material contrast. Peak force tapping is a non-resonant mode in which fast loaddisplacement curves are measured at each pixel in the image. With the proper calibrations, this mode provides several channels of information mapping stiffness (modulus), adhesion, dissipated energy, and deformation in addition to topography [24, 25]. For this work the same probe was used for both imaging methods and images collected on the same powder particle.

Both imaging methods were carried out on a MultiMode8 AFM using a Nanoscope V controller running v8.15 Nanoscope software (Bruker-Nano, CA). A silicon cantilever and tip (Arrow NCR, Nanoworld) was used. The tip radius was $11.2 \mathrm{~nm}$ determined by blind reconstruction 
using the TipCheck scanning artifact. The optical lever sensitivity of the AFM was determined to be $44.6 \mathrm{~nm} / \mathrm{V}$ using indentation on sapphire, and the spring constant of the cantilever was determined to be $46.7 \mathrm{~N} / \mathrm{m}$ using thermal tuning. The resonant frequency of the cantilever was $277 \mathrm{kHz}$. For the tapping mode, the cantilever free deflection was set to $500 \mathrm{mV}$, and the imaging set point was $400 \mathrm{mV}$ for a set point ratio of 0.80 . For the peak force tapping, the imaging was obtained with a set point of $100 \mathrm{nN}$, a peak force amplitude of $50 \mathrm{~nm}$ and a resonance frequency of $2 \mathrm{kHz}$. The tapping mode images and the peak force tapping were obtained at $512 \times 512$ pixels and $256 \times 256$ force curves, respectively. Selected force curves were analyzed using scanning probe image processor v6.35 (SPIP, Image Metrology, Denmark).

\subsection{Other Characterization}

The $\mathrm{pH}$ of the dispersion was measured using a Denver Instruments $\mathrm{pH}$ meter. The solid content of the dispersion was analyzed using an Ohaus MB35 series moisture analyzer. The dispersion particle size was analyzed on a Malvern Zetasizer Nano at $25.0 \pm 1{ }^{\circ} \mathrm{C}$, at a detection angle $173^{\circ}$ (backscattering), and the samples were diluted with deionized water and loaded into a disposable plastic cuvette.

The glass transition temperature $(\mathrm{Tg})$ was measured using a DSC 2920 differential scanning calorimeter from TA Instruments. Approximately $10 \mathrm{mg}$ of polymer was weighed into a DSC pan and then loaded into the instrument. The sample was cooled to $-40{ }^{\circ} \mathrm{C}$, held for $1 \mathrm{~min}$, and heated to $140{ }^{\circ} \mathrm{C}$ at a scan rate of $10{ }^{\circ} \mathrm{C}$ per min while measuring the heat flow. The midpoint of the heat flow transition was taken as the $\mathrm{Tg}$.

The epoxy content was titrated using an automated titrator (815 Robotic USB Sample Processor $\mathrm{XL})$. The desired amount of epoxy powder $(\sim 0.3 \mathrm{~g})$ was weighed out into a flask followed by the addition of $50 \mathrm{~mL}$ of methylene chloride. The solution was stirred with a magnetic stir bar until 
the epoxy powder was completely dissolved. Then, the solution was placed on the auto-sampler. Each sample was titrated at least twice to obtain consistent results.

\section{Results and Discussion}

This mechanical dispersion process has been applied to prepare aqueous dispersions of solid epoxy prepolymers with a wide range of anionic or non-ionic surfactants that maintain interfacial activity at high temperatures. PVOH is an effective dispersant to disperse epoxy prepolymers in water at elevated temperatures. Aqueous dispersions of solid epoxy prepolymers with wellcontrolled particle sizes have been successfully prepared [20]. In this work, we used a miscible blend of liquid epoxy prepolymer (D.E.R. ${ }^{\text {TM }}$ 331) and solid epoxy prepolymer (D.E.R. ${ }^{\text {TM }}$ 669E) to balance the physical properties of the epoxy phase. Liquid and solid epoxy prepolymers at a weight ratio of 45:55 were melt blended to form a homogenous semi-solid with a glass transition temperature of $23.1{ }^{\circ} \mathrm{C}$ based on the DSC profile in Figure 3. By using $10 \mathrm{wt} \%$ of PVOH based on the total epoxy solids, the resultant epoxy dispersion showed a mean average particle size of $346 \mathrm{~nm}$ by DLS. The $\mathrm{pH}$ of the epoxy dispersion was approximately 6.0.

In the previous methods for preparing epoxy-acrylic hybrid latexes [11-14] where liquid epoxy prepolymers were miscible with acrylic monomers, it was difficult to control the phase separation between the epoxy prepolymer and the acrylic polymer in the resulting latexes. Besides, liquid epoxy prepolymers are known to form a rigid but brittle thermoset after curing with amine agents. Despite good performance attributes of epoxy resin, it is often preferred to have low epoxy content in the hybrid latex particles to produce mechanically durable coatings. In comparison, high molecular weight epoxy prepolymers are capable of producing more flexible coatings upon crosslinking, and therefore, higher content of solid epoxy prepolymers in the 
hybrid particles can be beneficial. However, hybrid latex particles containing solid epoxy prepolymers have not been demonstrated in the literature, because solid epoxy prepolymers are not readily soluble in acrylic monomers and it is challenging to disperse them in water by using conventional emulsification techniques.

By our approach, the epoxy seed dispersion is prepared separately from the emulsion polymerization process, so the selection of epoxy prepolymers is not restricted by the emulsion polymerization process. We were interested in preparing epoxy-acrylic hybrid particles with a core-shell morphology. In the seeded polymerization process, we chose a copolymer composition of MMA and MAA at a 4:1 weight ratio and aimed at a shell to core ratio of 0.3. This acrylic polymer composition is also known as alkali soluble resin (ASR) [21] and it is insoluble under acidic conditions. The resultant hybrid dispersion has a $\mathrm{pH}$ value of 4.85 . The particle size of the latex samples were characterized by DLS, as shown in Figure 4. The particle size distribution of the epoxy-acrylic hybrid particles is mono-dispersed and consistently shifted to higher particle sizes, and more importantly, the particle size data shows that no acrylic particles formed in the seeded polymerization process. The mean particle diameter of the epoxyacrylic hybrid particles increases from 346 to $382 \mathrm{~nm}$, and the averaged shell thickness is approximately $18 \mathrm{~nm}$. Based on the weight increase of the epoxy-acrylic hybrid particles by $30 \%$, the calculated mean particle diameter is approximately $378 \mathrm{~nm}$ assuming both polymers have the same density, in good agreement with the measured particle size.

From the DSC profiles in Figure 2, two distinct $\mathrm{T}_{\mathrm{g}} \mathrm{s}$ suggest the epoxy and acrylic phases likely exhibit a phase-separated structure in the hybrid particles. The $T_{g}$ of the epoxy phase in the hybrid particles was $27.6{ }^{\circ} \mathrm{C}$, which slightly increased from the $\mathrm{T}_{\mathrm{g}}$ of the original epoxy prepolymers at $23.1{ }^{\circ} \mathrm{C}$, indicating a small amount of ASR polymer might be mixed into the 
epoxy-acrylic core shell structure was primarily attributed to kinetic trapping under the synthesis conditions. The monomer emulsion was gradually fed into the epoxy dispersion, where the monomer droplets were separated from the seed particles, so the epoxy phase was minimally plasticized by the monomers. During the seeded emulsion polymerization, the ASR oligomers were initially formed in the aqueous phase and migrated onto the surface of the epoxy particles. The ASR polymer could further diffuse into the epoxy phase, but it may require a higher energy due to the higher molecular weight solid epoxy prepolymer. Based on the AFM deformation images, few small ASR domains inside the epoxy phase prove that migration of the ASR oligomers into the epoxy phase indeed occurred during the seeded emulsion polymerization, but only to a relatively small extent.

In conventional seeded emulsion polymerization, chemical grafting is known as a critical factor that drives the formation of a core-shell morphology in hybrid polymer latexes [30], and the formation of new acrylic particles would be greatly suppressed in the seeded polymerization process. Herein, the grafting reactions between the epoxy and acrylic polymers likely drove the formation of the epoxy-acrylic core shell particles. There are two possible grafting mechanisms between the epoxy and acrylic polymers in the literature: the reaction between carboxylic acids and epoxy groups [31] and radical grafting onto epoxy aliphatic carbon [32]. The epoxy groups were titrated using a standard method to show an epoxy equivalent weight (EEW) of 386.6 gram/equivalent; meanwhile, the total EEW was calculated to be 388.7 gram/equivalent based on the epoxy phase. The minor change in the EEW suggests that the reaction of the epoxy groups and carboxylic acid was insignificant. However, neither of grafting reactions will cause significant changes in the epoxy EEW because the reactions occur predominantly at the 
interface. We will have to further analyze the chemical structure of the epoxy-acrylic hybrid particles to distinguish the above two mechanisms in future work.

\section{Conclusions}

We have demonstrated the synthesis of novel epoxy-acrylic hybrid latexes by a combination of the mechanical dispersion and traditional emulsion polymerization processes. The resulting hybrid particles show a unique core-shell morphology by both STXM and PFT-AFM images. We were able to control the epoxy and acrylic polymers in two independent steps. We can potentially achieve a broad range of epoxy and acrylic compositions in the hybrid particles, as compared with the previous literature methods. For the epoxy-acrylic core-shell particles with a high $T_{g}$ shell, the well-defined acrylic shell provides the low $T_{g}$ epoxy core with good protection against irreversible coalescence upon drying, which can potentially lead to new applications of the epoxy-acrylic hybrid particles as powder additives [33]. This approach may also be applied to design epoxy-acrylic hybrid particles with different morphologies. Moreover, this new approach has shed some light on a new strategy for designing a broad range of novel hybrid particles beyond using the conventional emulsion polymerization technique.

\section{ACKNOWLEDGMENTS}

We thank Ben Schaefer for help with the epoxy titration experiment. We also thank Susan Fitzwater for valuable discussions and Tom Kalantar for helpful suggestions. The authors thank A.L.D. Kilcoyne for beamline support throughout the study and for general discussions of the beamline and microscope. STXM data acquired at beamline 5.3.2.2 at the Advanced Light 
Source, Berkeley, which is supported by the Director, Office of Science, Office of Basic Energy

Sciences, of the U.S. Department of Energy under Contract No. DE-AC02-05CH11231.

\section{REFERENCES}

[1] D.C. Sundberg, A.P. Casassa, J. Pantazopoulos, M.R. Muscato, Morphology Development of Polymeric Microparticles in Aqueous Dispersions. I. Thermodynamic Considerations, J. Appl. Polym. Sci. 14 (1990) 1425-1442.

[2] S.C. Thickett, R.G. Gilbert, Emulsion polymerization: State of the Art in Kinetics and Mechanisms, Polymer 48 (2007) 6965-6991.

[3] F. Jahanzad, E. Karatas, B. Saha, B.W. Brooks, Hybrid Polymer Particles by Miniemulsion Polymerization, Colloids Surf. A: Physicochem. Eng. Asp. 302 (2007) 424-429.

[4] A. Guyot, K. Landfester, F.J. Schork, C. Wang, Hybrid Polymer Latexes, Prog. Polym. Sci. 32 (2007) 1439-1461.

[5] C.-Y. Kan, X.-Z. Kong, Q. Yuan, D.-S. Liu, Morphological Prediction and Its Application to the Synthesis of Polyacrylate/Polysiloxane Core/Shell Latex Particles, J. Appl. Polym. Sci. 80 (2001) 2251-2258.

[6] M. Hirose, J. Zhou, K. Nagai, The Structure and Properties of Acrylic-Polyurethane Hybrid Emulsions, Prog. Org. Coating 38 (2000) 27-34.

[7] I. Cho, K.-W. Lee, Morphology of Latex Particles Formed by Poly(methyl methacrylate)Seeded Emulsion Polymerization of Styrene, J. Appl. Polym. Sci. 30 (1985) 1903-1926.

[8] K.A. Wood, Optimizing the Exterior Durability of New Fluoropolymer Coatings, Prog. Org. Coating 43 (2001) 207-213. 
[9] R.A. Ramli, W.A. Laftah, S. Hashim, Core-Shell Polymers: A Review, RSC Adv. 3 (2013) 15543-15565.

[10] K.D. Weiss, Paint and Coatings: A Mature Industry in Transition, Prog. Poly. Sci. 22 (1997) 203-245.

[11] G.L. Brown, M.A. Tobias, Epoxy Modified Acrylic Lattices and Method of Producing Same, U.S. Patent 4028294, 1977.

[12] H. Kawahara, T. Goto, K. Ohnishi, H. Ogura, H. Kage, Y. Matsuno, Preparation of Epoxy Resin/Acrylic Composite Latexes by Miniemulsion Polymerization Method, J. Appl. Polym. Sci. 81 (2001) 128-133.

[13] G. Pan, L. Wu, Z. Zhang, D. Li, Synthesis and Characterization of Epoxy-Acrylate Composite Latex, J. Appl. Polym. Sci. 83 (2002) 1736-1743.

[14] L. Liu, J. Li, X. Zhang, K. Jin, The Preparation of a Three-layer "Core-Shell” Structured Epoxy-Acrylate Emulsion, RSC Adv. 4 (2014) 47184-47190.

[15] G.W. Dombrowski, Z. Fu, C. Slone, A. Swartz, Epoxy Resin Imbibed Polymer Particles, U.S. Patent 8658742, 2014.

[16] N.R. Cameron, D.C. Sherrington, High Internal Phase Emulsions (HIPEs) - Structure, Properties and Use in Polymer Preparation, Adv. Polym. Sci. 126 (1996) 163-214.

[17] J.E. Pate, J. Peters, N.E. Lutenske, R.R. Pelletier, Process for Preparing High Internal Phase Ratio Emulsions and Latexes Derived Thereof, U.S. Patent 5539021, 1996.

[18] C.F. Welch, G.D. Rose, D. Malotky, S.T. Eckersley, Rheology of High Internal Phase Emulsions, Langmuir 22 (2006) 1544-1550.

[19] H.Q. Pham, M.J. Marks, Epoxy Resins in Encyclopedia of Polymer Science and Technology; Mark, H. F. Ed.; John Wiley \& Sons, Inc., 2004. 
[20] L. Chen, L. Hong, An Epoxy Resin Blend Dispersion and a Process for Preparing the Dispersion, U.S. Patent Appl. 20150105492A1, 2015.

[21] D.P. Lorah, Grafted Core-Shell Polymer Compositions Using Polyfunctional Compounds. U.S. Patent 4876313, 1989.

[22] A.L.D. Kilcoyne, T. Tyliszczak, W.F. Steele, S. Fakra, P. Hitchcock, K. Franck, E. Anderson, B. Harteneck, E.G. Rightor, G.E. Mitchell, A.P. Hitchcock, L. Yang, T. Warwick, H. Ade, Interferometer-controlled Scanning Transmission X-Ray Microscopes at the Advanced Light Source, J. Synchrotron Rad. 10 (2003) 125-136.

[23] Axis is freeware written by Professor Adam Hitchcock at McMaster University and is described and available for download at the following link: http://unicorn.mcmaster.ca/aXis2000.html.

[24] C. Su, Mapping Quantitative Mechanical Properties at Molecular Scale Using Peak Force Tapping AFM, Microscopy and Microanalysis 16 (2010) 364-365.

[25] P. Schon, K. Bagdi, K. Molnar, P. Markus, B. Pukanszky, G.J. Vancso, Quantitative Mapping of Elastic Moduli at the Nanoscale in Phase Separated Polyurethanes by AFM, Eur. Polym. J. 47 (2011) 692-698.

[26] J.A. Forrest, K. Dalnoki-Veress, The Glass Transition in Thin Polymer Films, Adv. Colloid Interface Sci. 94 (2001) 167-196.

[27] H. Ade, A.P. Hitchcock, NEXAFS Microscopy and Resonant Scattering: Composition and Orientation Probed in Real and Reciprocal Space, Polymer 49 (2008) 643-675.

[28] H. Ade, S. Urquhart, NEXAFS Spectroscopy and Microscopy of Natural and Synthetic Polymers in Chemical Applications of Synchrotron Radiation; Sham, T.K., Ed.; World Scientific Publishing Company: River Edge, NJ, 2002. 
[29] S. Ritzenthaler, E. Girard-Reydet, J.P. Pascault, Influence of Epoxy Hardener on Miscibility of Blends of Poly(methyl methacrylate) and Epoxy Networks, Polymer 41 (2000) $6375-6386$.

[30] T.I. Min, A. Klein, M.S. El-Aasser, J.W. Vanderhoff, Morphology and Grafting in Polybutylacrylate-Polystyrene Core-Shell Emulsion Polymerization, J. Polym. Sci.: Polym. Chem. 21 (1983) 2845-2861.

[31] L. Shechter, J. Wynstra, Glycidyl Ether Reactions with Alcohols, Phenols, Carboxylic Acids, and Acid Anhydrides, Ind. Eng. Chem. 48 (1956) 86-93.

[32] J.T.K. Woo, A. Toman, Water-based Epoxy-Acrylic Graft Copolymer, Prog. Org. Coat. 21 (1993) 371-385.

[33] L. Chen, L. Hong, M. Sekharan, M. Radler, Epoxy RDP and Method for Preparing Same. U.S. Patent 9200155, 2015. 


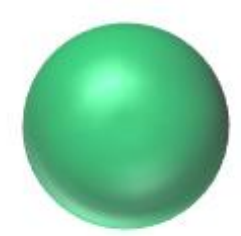

Epoxy Dispersion

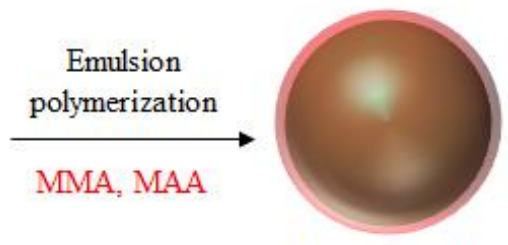

Epoxy-acrylic core-shell latex
Epoxy prepolymer:

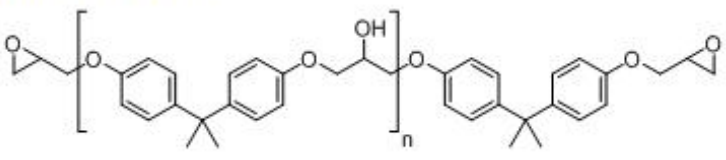

Acrylic polymer:

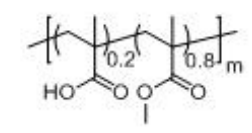

Figure 1. Preparation of epoxy-acrylic core-shell particles via a seeded emulsion polymerization process.

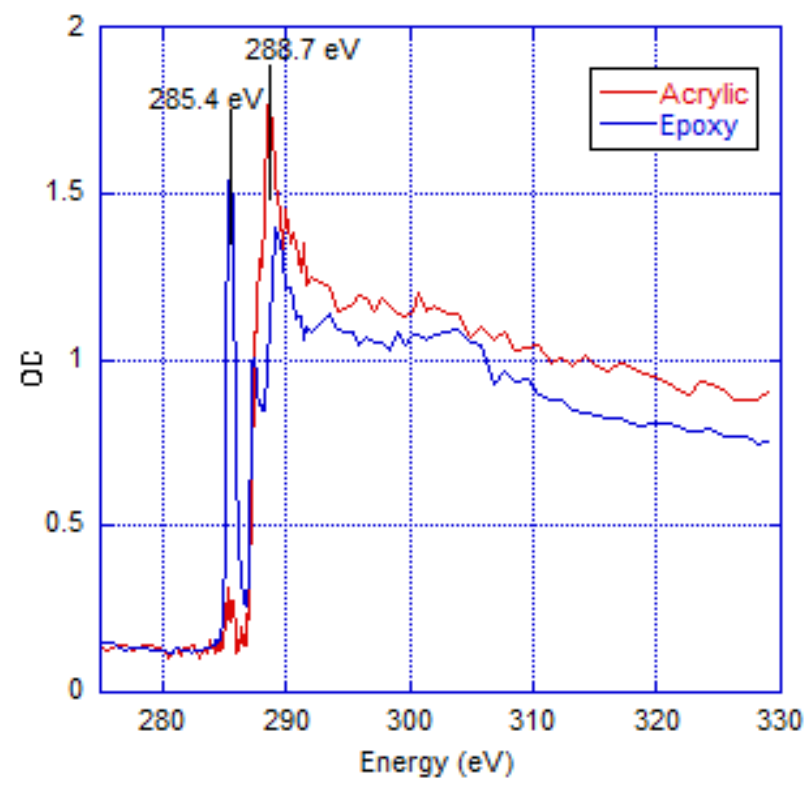

Figure 2. The NEXAFS spectra of the epoxy prepolymers and the acrylic polymer. 


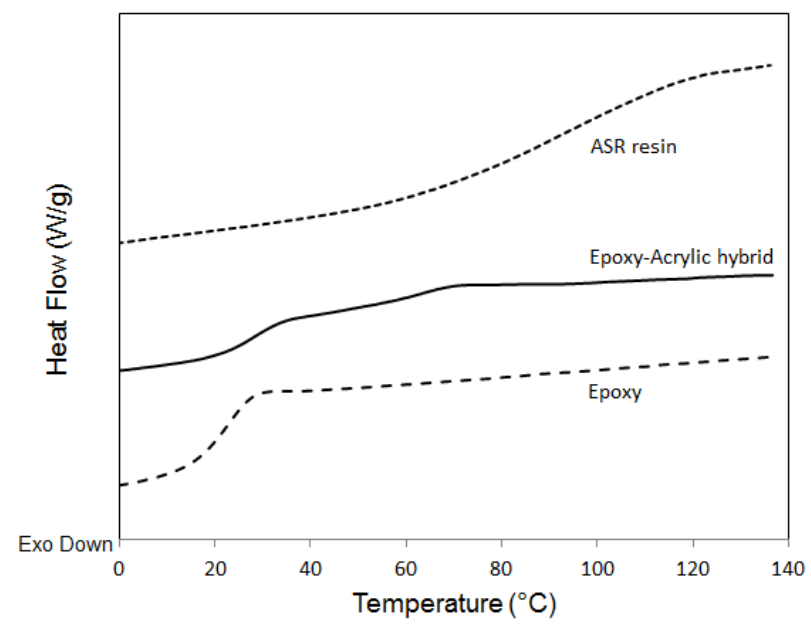

Figure 3. The DSC traces of the epoxy prepolymers, the epoxy-acrylic hybrid particles, and the ASR resin. The signals are shifted vertically.

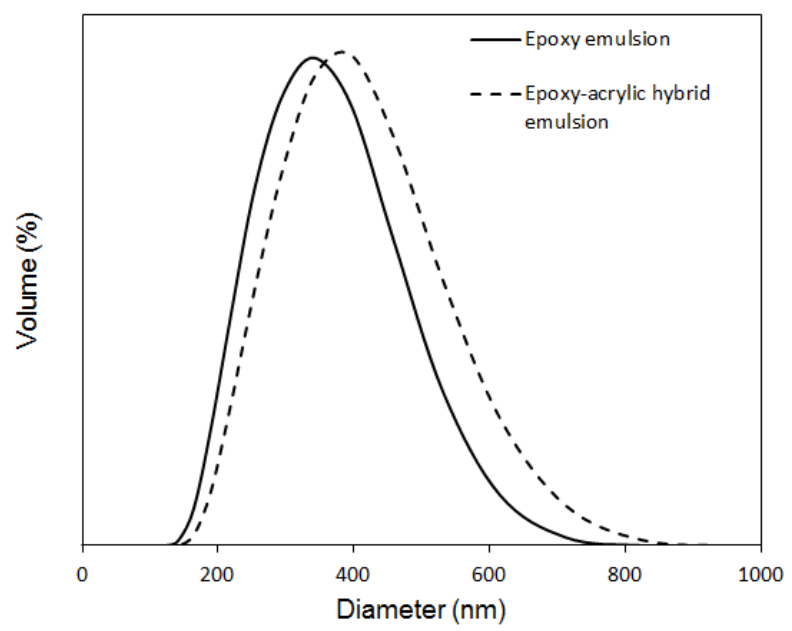

Figure 4. The particle size distribution curves of the epoxy dispersion and the epoxy-acrylic hybrid latex with $30 \%$ ASR. 


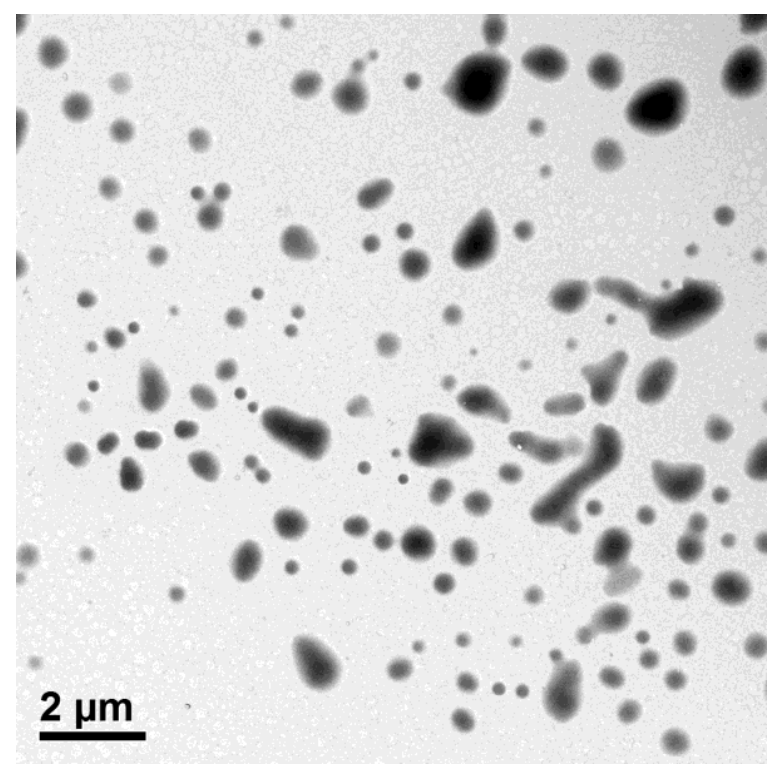

(a)

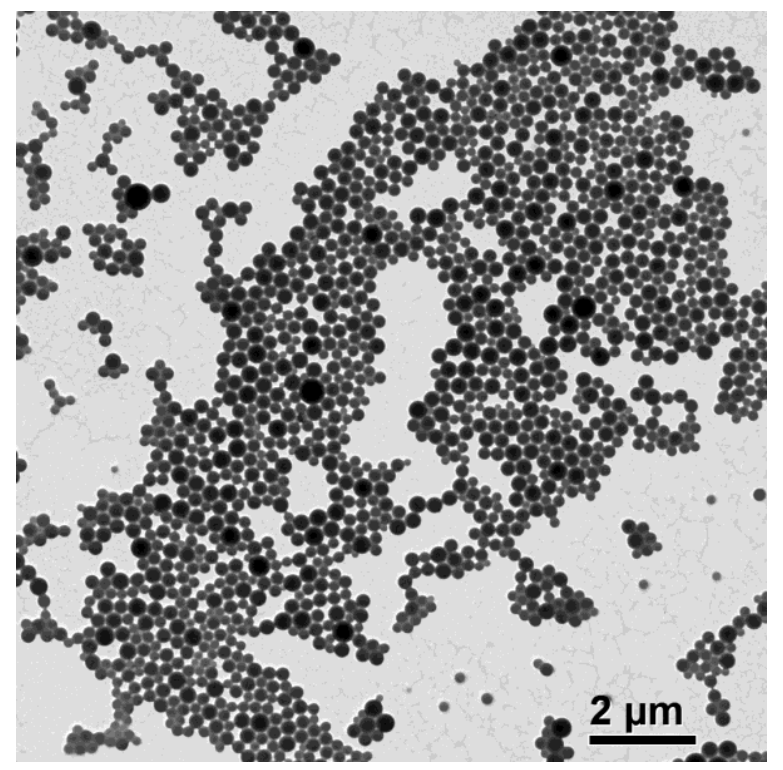

(b)

Figure 5. The TEM images of the epoxy particles (a) and the epoxy-acrylic hybrid particles (b). 

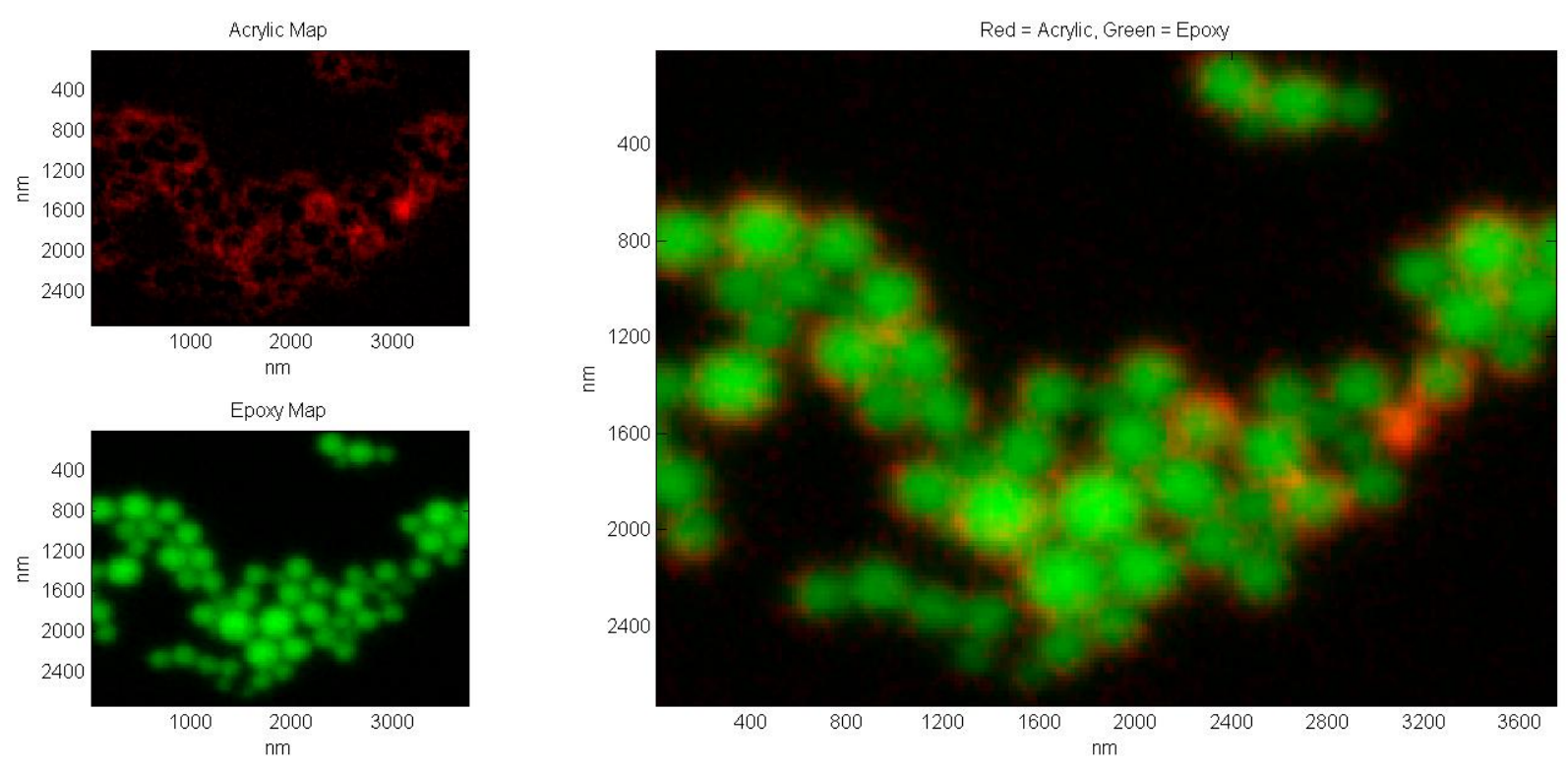

Figure 6. An STXM image of the epoxy-acrylic hybrid particles with $30 \%$ ASR (MMA:MAA=4:1). 


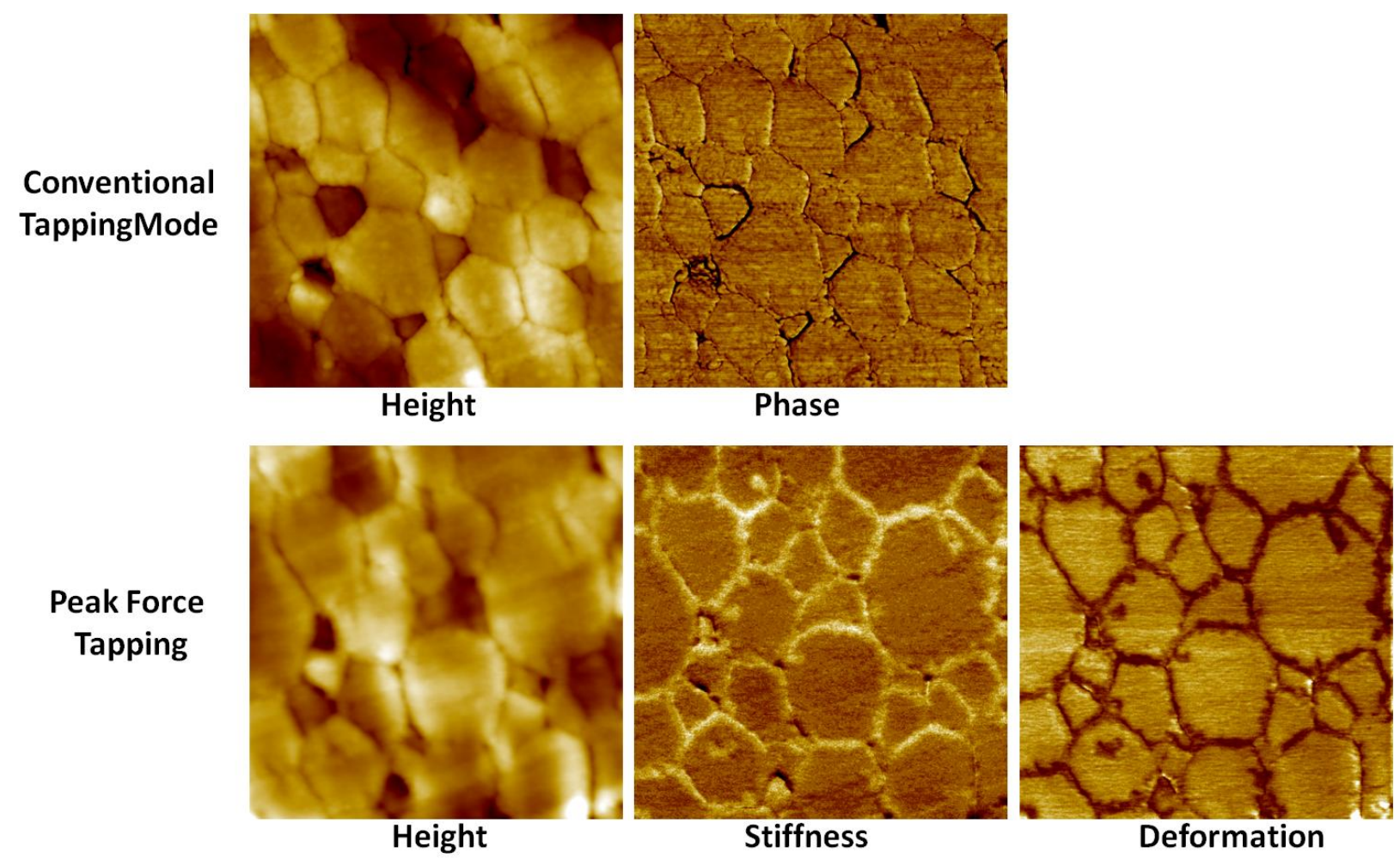

Figure 7. The AFM images of the epoxy-acrylic hybrid particles (image $1.5 \mu \mathrm{m} \times 1.5 \mu \mathrm{m}$ ). 


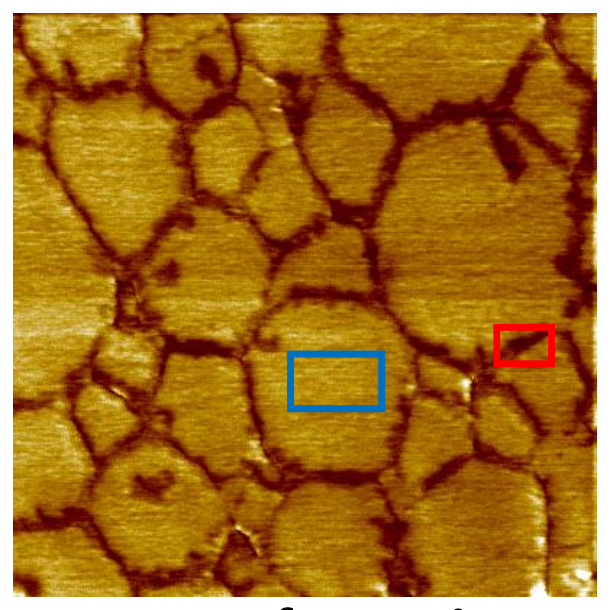

Deformation

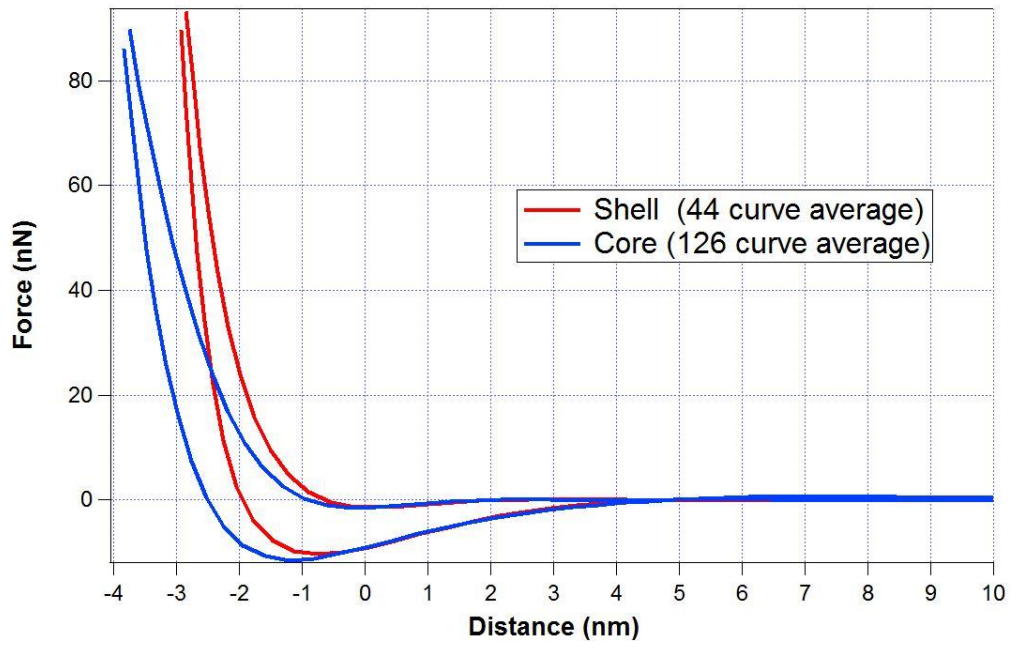

Figure 8. Force curve analysis from PFT. Curves from representative core (blue) and shell (red) regions of the morphology were averaged and analyzed; in this case, they are shown in the deformation map (left). Average force vs. distance curves are compared (right). The curves are discontinuous at the peak force because they are averages. The experimental peak force was 100 $\mathrm{nN}$. 


\section{STXM images of epoxy-acrylic core-shell latex}

\section{Epoxy Map}

\section{Acrylic Map}
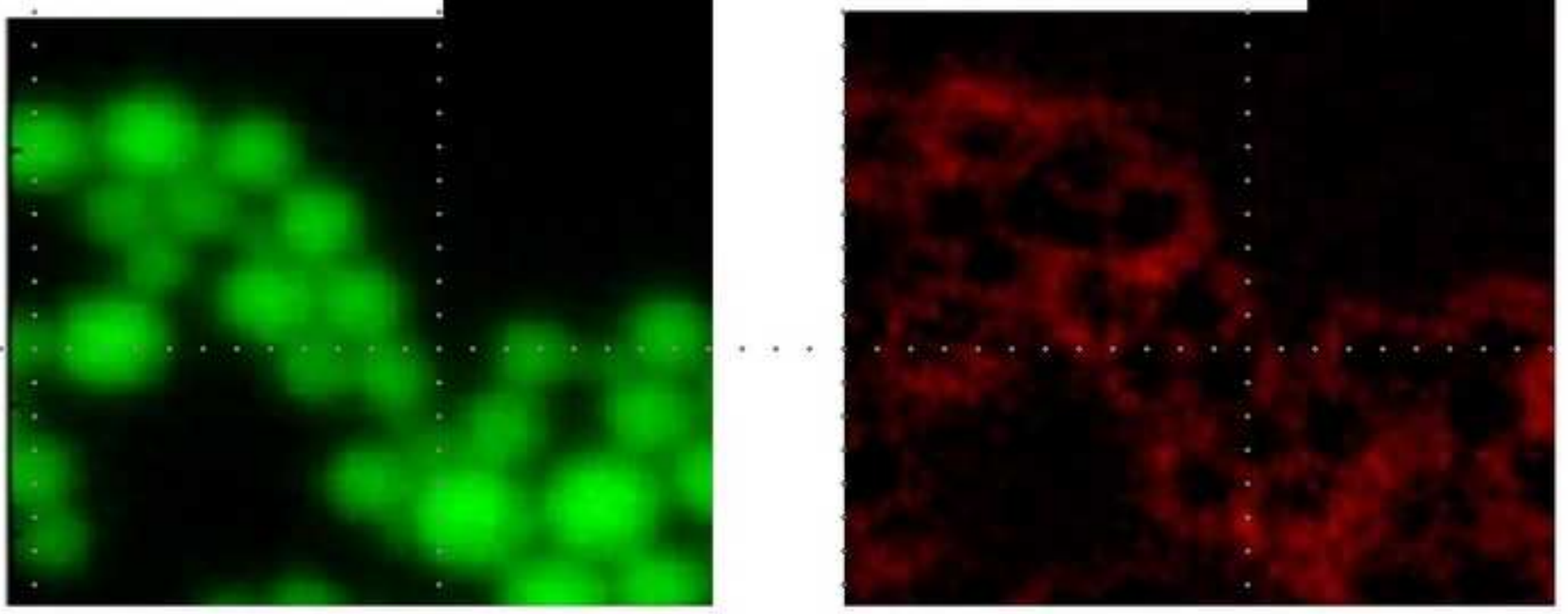